\title{
Influence of Island Scanning Strategy on Microstructures and Mechanical Properties of Direct Laser-Deposited Ti-6AI-4V Structures
}

\author{
Xiao Wang $^{1} \cdot$ Fei Lv $^{1} \cdot$ Li-Da Shen $^{1} \cdot$ Hui-Xin Liang ${ }^{1} \cdot$ De-Qiao Xie $^{1} \cdot$ Zong-Jun Tian $^{1}$ \\ Received: 28 August 2018 / Revised: 11 October 2018 / Published online: 5 December 2018 \\ (c) The Chinese Society for Metals (CSM) and Springer-Verlag GmbH Germany, part of Springer Nature 2018
}

\begin{abstract}
To investigate the influence of island scanning on the microstructures and mechanical properties of direct laser-deposited Ti-6Al-4V structures, two samples are prepared using island scanning and orthogonal successive scanning, respectively. The microstructures, relative density, and mechanical properties of the samples prepared using these two scanning strategies are compared. Each sample exhibits columnar $\beta$-grain morphology and basket-weave microstructure characterization. The grains of the sample prepared using island scanning are significantly finer than that prepared by orthogonal successive scanning due to faster cooling during deposition. However, the relative density of the sample prepared using island scanning was slightly smaller due to the concentration of lack-of-fusion pores at the overlap zone of the island. Tensile testing at room temperature indicates that the ultimate tensile strength and yield strength of the sample prepared using island scanning is enhanced due to finer grains, while the ductility of the sample is weakened due to defects.
\end{abstract}

Keywords Direct laser deposition $\cdot \mathrm{Ti}-6 \mathrm{Al}-4 \mathrm{~V} \cdot$ Scanning strategies $\cdot$ Microstructure $\cdot$ Defects $\cdot$ Mechanical property

\section{Introduction}

Direct laser deposition (DLD) is an advanced laser-based additive manufacturing technology for fabricating metal components with full density, any geometry, and high performance [1-3]. During DLD, metal powder is injected continuously into the molten pool, created using a high-powered laser, on the surface of the workpiece. With the movement of the laser or substrate following the user-defined path, threedimensional metal parts can be formed point by point, line by line, and layer by layer. Because of the capability of DLD to fabricate near-net-shape components without substantial machining, it creates less material waste and a shorter lead time $[4,5]$. Therefore, the method has become increasingly

Available online at http://link.springer.com/journal/40195

Xiao Wang and Fei Lv have contributed equally to this work.

Zong-Jun Tian

tianzj@nuaa.edu.cn

1 College of Mechanical and Electrical Engineering, Nanjing University of Aeronautics and Astronautics, Nanjing 210016, China attractive for fabricating expensive titanium aerospace components. Unfortunately, two key issues still hinder the development of this technology: the relatively large thermal stress produced during the formation process $[6,7]$, and the control of the microstructure and mechanical stability of the samples $[8,9]$.

The island scanning strategy has been gradually applied in the formation process of large structures [10]. The island scanning strategy, a patented scanning pattern from Concept Laser, is the stochastic exposure strategy in line with the “island principle." In order to decrease the residual stresses, each layer is divided into smaller islands, and these islands are raster scanned with shorter scan tracks in a random order. The vectors in the neighboring islands are perpendicular to each other. There are different island scanning strategies with the islands of different sizes and different island scanning orders. Structural warpage and cracking can be suppressed with this scanning strategy. However, the microstructure and mechanical properties of structures prepared by island scanning have yet to be investigated. Previous studies have shown that the microstructural characteristics of DLD parts are strongly sensitive to their thermal history during the building process [11-13]. Laser scanning can influence heat input and heat dissipation during building, 
further influencing the evolution of the microstructure of DLD parts [14-16]. Therefore, to control the microstructure and mechanical properties of large structures, it is essential to study the difference in microstructure and mechanical properties of the samples prepared using island scanning and other scanning strategies.

In this study, island scanning and orthogonal successive scanning are used to prepare Ti-6 $\mathrm{Al}-4 \mathrm{~V}$ samples by direct laser deposition. The influence of island scanning on the microstructure and mechanical properties of direct laserdeposited $\mathrm{Ti}-6 \mathrm{Al}-4 \mathrm{~V}$ structures is investigated.

\section{Experimental}

Experiments were performed on a DLD system consisting of a $4 \mathrm{~kW}$ semiconductor laser (LDF, Laserline), a three-axis Computerized Numerical Control unit (Siemens), a powder feeder, and a 4-beam nozzle. Laser energy was delivered through a $1000 \mu \mathrm{m}$ diameter optical fiber into a $5 \mathrm{~mm}$

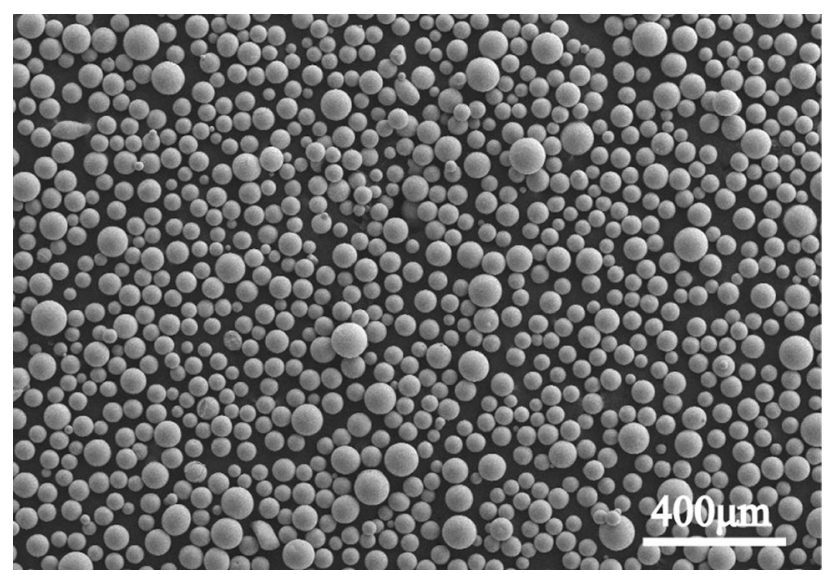

Fig. 1 SEM image of morphology of Ti-6Al-4V powders diameter focal point that generates a melt pool. Powder was fed by argon gas through a 4-beam nozzle into the melt pool.

Ti-6Al-4V alloy powder prepared by the plasma rotation electrode process (PREP) in the size range of 45-105 $\mu \mathrm{m}$ was deposited. The scanning electron microscope (SEM) image in Fig. 1 shows that most powders are spherical. The chemical composition of Ti-6Al-4V alloy powder is as follows (wt\%): $6.02 \mathrm{Al}, 4.00 \mathrm{~V}, 0.15 \mathrm{Fe}, \leq 0.039 \mathrm{Si}, \leq 0.056 \mathrm{C}$, $0.12-0.16 \mathrm{O}, 0.046 \mathrm{~N}, 0.01 \mathrm{H}$ and balance Ti. A Ti-6Al-4V alloy sheet was used as substrate. Based on our previous experimental study, the laser power is $2500 \mathrm{~W}$, the scanning velocity is $720 \mathrm{~mm} / \mathrm{min}$, the powder feeding rate is $7 \mathrm{~g} / \mathrm{min}$ and the overlap is $50 \%$. All fabrication experiments were conducted in an argon gas-filled pressurized box with $\mathrm{O}_{2}$ strictly controlled below $50 \mathrm{ppm}$.

First, two typical groove structures with identical dimensions were prepared using island scanning and orthogonal successive scanning, respectively. The dimensions of the groove structures are shown in Fig. 2a. Each groove structure consists of three walls with the same height and thickness but different lengths. The orthogonal successive scanning strategy indicates that every layer of the single wall is deposited successively by laser. The laser scanning path is parallel to the long side when old layers are deposited and parallel to the short side when even layers are deposited. The laser scanning path on the even layer is shown in Fig. 2b. In contrast, with the island scanning strategy, every deposited layer is divided into several same-sized islands, and then the islands are deposited in a random order to mitigate continuous local heat accumulation. In this experiment, the size of the divided single island was $20 \mathrm{~mm} \times 20 \mathrm{~mm}$, and the length of the island was the same as the thickness of the wall, which is shown in Fig. 2c. To ensure lap quality, the scanning vectors between adjacent layers or islands were perpendicular to each other, and the processing parameters were the same for the two scanning strategies.

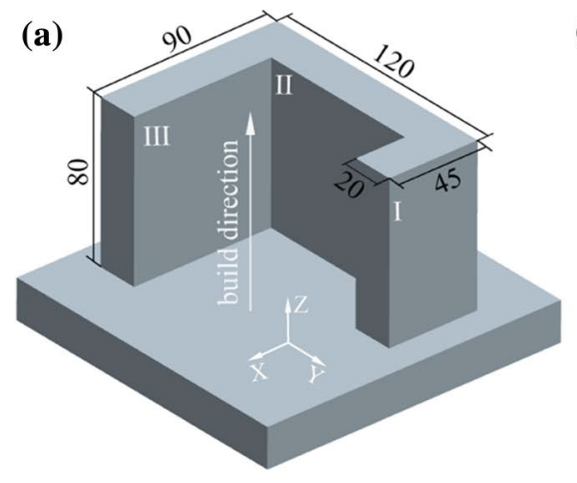

(b)

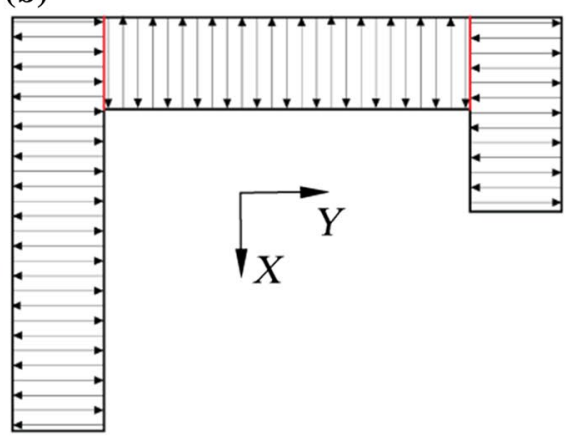

(c)

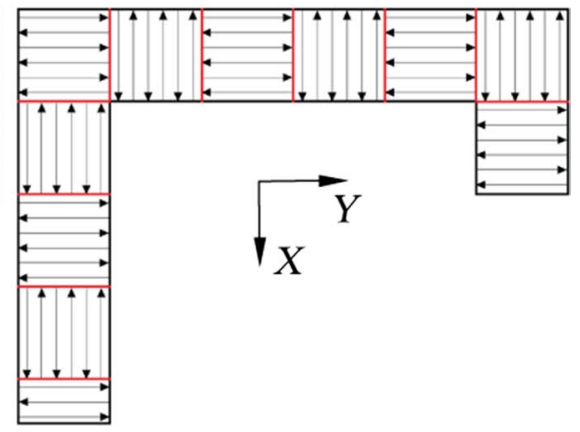

Fig. 2 Direct laser-deposited Ti-6Al-4V structures (mm): a build dimension; b laser scanning path of orthogonal successive scanning on even layers; $\mathbf{c}$ laser scanning path of island scanning 
Wall II of the structures formed by island scanning and orthogonal successive scanning strategies, respectively, were sectioned in the $X-Z$ plane for microstructure observation. The microstructure pieces had the same position in each structure for comparison purposes. Metallographic samples were prepared by mechanical polishing. A chemical solution of $1 \mathrm{ml} \mathrm{HF}, 2 \mathrm{ml} \mathrm{HNO}_{3}$, and $15 \mathrm{ml} \mathrm{H}_{2} \mathrm{O}$ served as the etching agent. Then, the microstructures of the samples were characterized using optical microscopy (ZEISS Lab.A1).

Next, the relative density of the structures using the two scanning strategies was examined and compared. The relative density was measured by the drainage method. Three pieces were sectioned across a transverse direction from each structure for measurement, with pieces taken from consistent positions in both structures. Each piece was measured three times to get an average. An electronic balance was adopted as the weighing instrument. The measurement precision of the balance is $0.1 \mathrm{mg}$, which can provide accurate measurement. Furthermore, due to defects in the main factors affecting density [11], the interior quality of the samples prepared using the two scanning strategies were examined by optical microscopy. The island overlap of the structure prepared via island scanning was expected to have concentrated defects and was therefore observed more closely.

Finally, the mechanical properties of the structures prepared using the two scanning strategies were analyzed. Tensile testing was performed at room temperature on an ETM205D testing machine with a constant crosshead displacement rate of $1 \mathrm{~mm} / \mathrm{min}$. A tensile test was carried out according to the test standard of GB/T 228-2002. Horizontal and vertical dog-bone shaped specimens from wall II and wall III of each structure were tested and compared. Then, the fracture morphology of the tensile samples was characterized using SEM (Hitachi S-4800N).

\section{Results and Discussion}

\subsection{Microstructure Characterization}

The macrostructure and microstructure of the samples are shown in Fig. 3. Columnar prior $\beta$ grains, which grow through multiple layers along the build direction, appear in both samples as shown in Fig. 3a, b. The columnar $\beta$
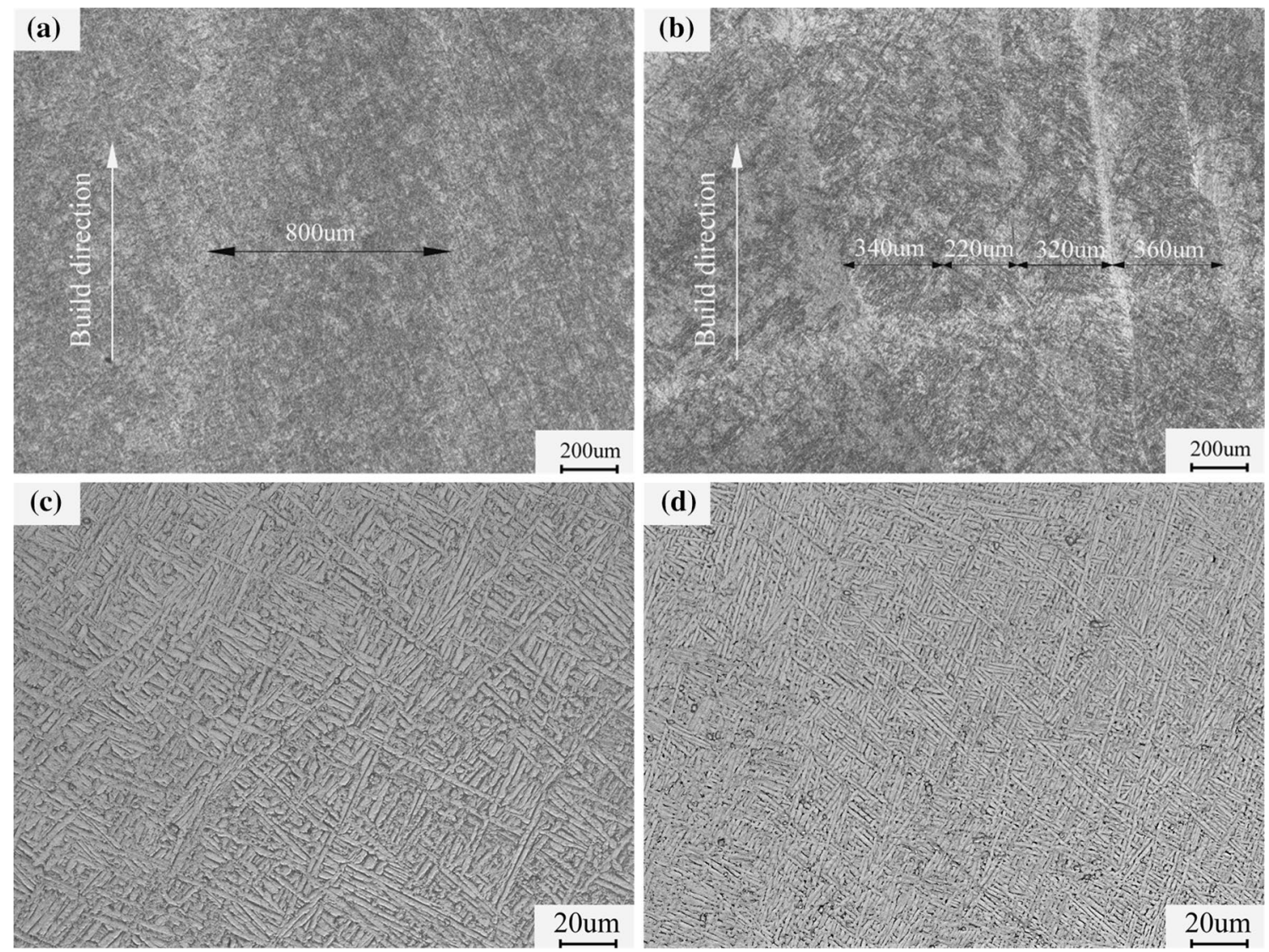

Fig. 3 Macrostructure and microstructure of samples: a macrostructure by orthogonal scanning; $\mathbf{b}$ macrostructure by island scanning; $\mathbf{c}$ intragranular $\alpha$ phase feature by orthogonal scanning; $\mathbf{d}$ intragranular $\alpha$ phase feature o by island scanning 
grains occur due to a high positive temperature gradient from the bottom to the top of the molten pool during the solidification process. However, the width of $\beta$ grains in the samples prepared using different scanning strategies are distinct. The width of $\beta$ grains is about $800 \mu \mathrm{m}$ for the sample prepared by orthogonal successive scanning but only ranges from 220 to $360 \mu \mathrm{m}$ for the sample prepared using island scanning. Furthermore, the intragranular $\alpha$-phase feature shown in Fig. 3c, d exhibits a basketweave microstructure characterization within the $\beta$ grains in both samples. Similarly, the size of the $\alpha$ laths is different in each sample: the average $\alpha$ lath width in the sample prepared using island scanning is approximately $1 \mu \mathrm{m}$, half that in the sample prepared by orthogonal successive scanning. Moreover, the island scanning sample exhibits a smaller length-to-width ratio for $\alpha$ laths. Therefore, the island scanning strategy can effectively refine the microstructure in the sample.

When Ti-6Al-4V undergoes $\beta$ - to $\alpha$-phase transformation during solidification, different cooling rates produce different morphologic forms in the $\alpha+\beta$ phase [2]. The grains also tend to be finer when the cooling rate is faster. To determine why island scanning refined the grains in the sample, it is necessary to compare the thermal history of the orthogonal successive scanning and island scanning strategies. With the continuous deposition process, the temperature of the deposited layers tends to remain stable, and the temperature gradient of the scanning nodes is generally consistent with both scanning strategies. However, each wall of the sample prepared using orthogonal successive scanning undergoes continuous heat accumulation, leading to a relatively high temperature base for the subsequent cladding channel prior to laser treatment. Therefore, when the channel is deposited completely and the laser is removed, channel overcooling is relatively slight. As for island scanning, when each divided island is small enough, the scanning duration per island is quite short; thus, heat accumulation is minimized, and the temperature base of the subsequent cladding channel is reduced before laser treatment. Yet in the process of random jumps between islands, the continuous heat input from subsequent deposition to the deposited cladding channels is interrupted. Two factors increase the cooling rate of the deposited cladding channels; therefore, the $\alpha$ phase nucleation rate in different directions is increased, and the size of the $\alpha$ laths is significantly reduced by island scanning compared with orthogonal successive scanning. Similarly, as in orthogonal successive scanning, the subsequent deposition process exerts various heating and insulation effects on the deposited channels that have completed phase transformation (whether in odd or even layers), leading to secondary growth of columnar $\beta$ grains and a larger columnar $\beta$-grain width. Overall, island scanning is beneficial to refine grains in the sample whether they are $\beta$ or $\alpha$ grains.

\subsection{Relative Density and Defects}

The three samples prepared by island scanning exhibit a lower relative density compared with those prepared using orthogonal successive scanning, as shown in Fig. 4. After calculation, the average relative density of the samples prepared with island scanning and orthogonal successive scanning are $98.92 \%$ and $99.66 \%$, respectively. The difference between them is quite small, and the relative density values are both sufficiently high, indicating that both strategies can form high-density parts.

Figure 5 displays the optical micrographs of the direct laser-deposited samples using the two scanning strategies. Because island scanning forms the part area by area and then connects them, the hot-and-cold alternation of the island junction is complex, increasing the potential for defect formation. Therefore, the longitudinal section of island overlap in the sample prepared using island scanning is examined and compared with the same position in the sample prepared by orthogonal successive scanning. As shown in Fig. 5a, c, the sample prepared by orthogonally successive scanning shows high interior quality with no obvious defects. The island scanning sample shows defect aggregation in the island overlap, as shown in Fig. 5b, d; the defect size is generally $40-45 \mu \mathrm{m}$. These results indicate that island scanning tends to introduce more defects because more island overlap occurs during the process, concurring with the density test results.

Upon further examining the defect morphology, the defects exhibit irregular shapes, unlike the spherical pores that generally arise from gas discharge in direct laser deposition. These defects are known as lack-of-fusion pores. Notably, the overlap width between the islands adopted in the experiment is sufficiently large; thus, lack-of-fusion pores are unlikely to be caused by bad lapping. By analyzing the

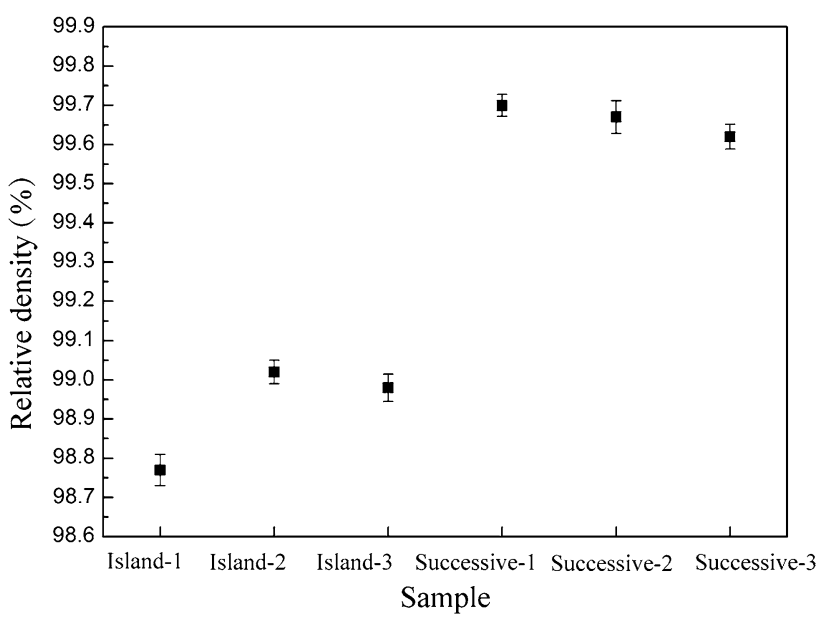

Fig. 4 Relative density of samples by different scanning strategies 

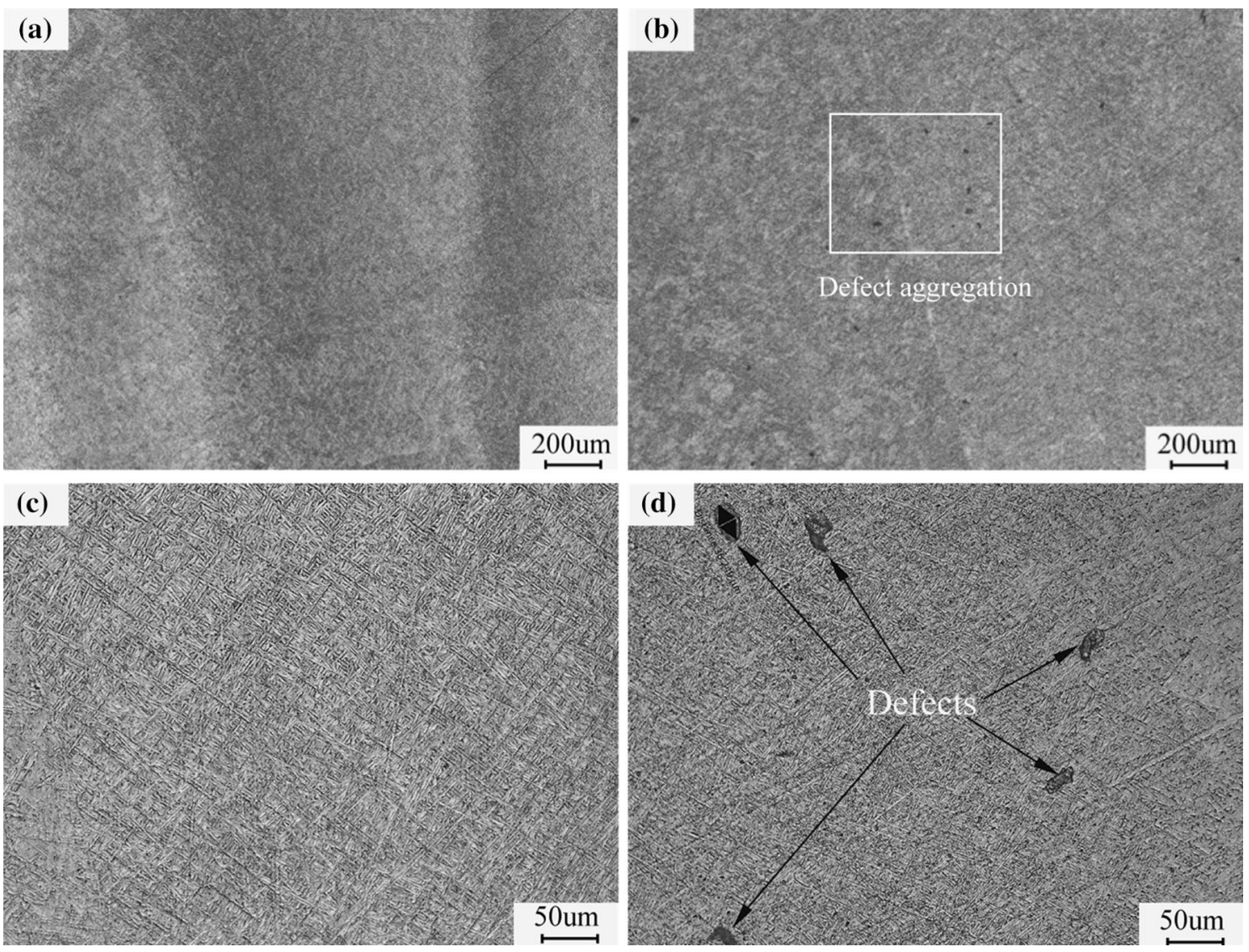

Fig. 5 Interior quality of direct laser-deposited samples by different scanning strategies: a, c orthogonal successive scanning; b, $\mathbf{d}$ island scanning

island scanning process, we believe that the lack-of-fusion pores are mainly related to the vertical overlap between the scanning lines in the adjacent islands. As shown in Fig. 6, in orthogonal successive scanning, the overlap of the adjacent cladding channels all occurs on the long side; thus, the lap surface is smooth. With island scanning, however, adjacent islands are connected by overlapping from the ends of the cladding channels in one island to the long side of the cladding channel in the other. Even if the deposition process is not interrupted at the island edges, the scanning process must still undergo acceleration and deceleration switching, thus causing the uneven edges in the newly deposited island and resulting in residual powders. In the subsequent layer deposition, due to the surface tension of the molten pool, the depressed position of the overlap cannot be completely filled by the molten pool, leading to lack-of-fusion defects.

\subsection{Mechanical Properties}

Figure 7 shows the stress-strain curves of the horizontal and vertical specimens sectioned from structures using the two scanning strategies in tensile testing at room temperature. Accurate values of the main tensile properties are shown in Table 1, indicating that ultimate tensile stress and yield strength of the island-scanned samples are higher than those prepared by orthogonal successive scanning. The ductility of the island-scanned vertical sample is also higher than that scanned using the orthogonal successive method; however, the ductility of the horizontal samples shows the opposite pattern.

These experiments indicate that island scanning is beneficial for grain refinement, but it introduces more lack-offusion defects, which has important impact on the tensile properties of the formed parts. According to the Hall-Petch formula [17], with the island scanning strategy, finer $\alpha$ grains in the sample produce more $\alpha$ grain boundaries and block dislocation slip, thus increasing the yield strength of the sample. Therefore, both the horizontal and vertical samples prepared by island scanning exhibit larger yield strength and ultimate strength compared with those prepared using orthogonal successive scanning. However, the existence of internal defects still affects the plastic properties of the island-scanned samples. Despite showing nearly no difference in elongation, the plastic instability point appears earlier on the stress-strain curve of the horizontal sample prepared by island scanning, and its uniform elongation is 
(a)

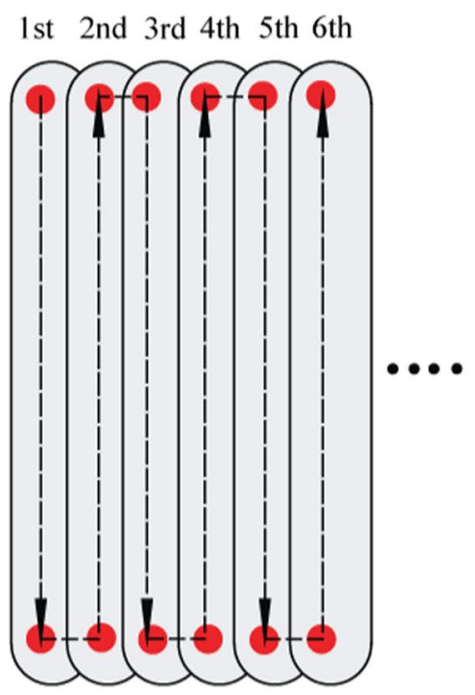

(b)

Island overlap

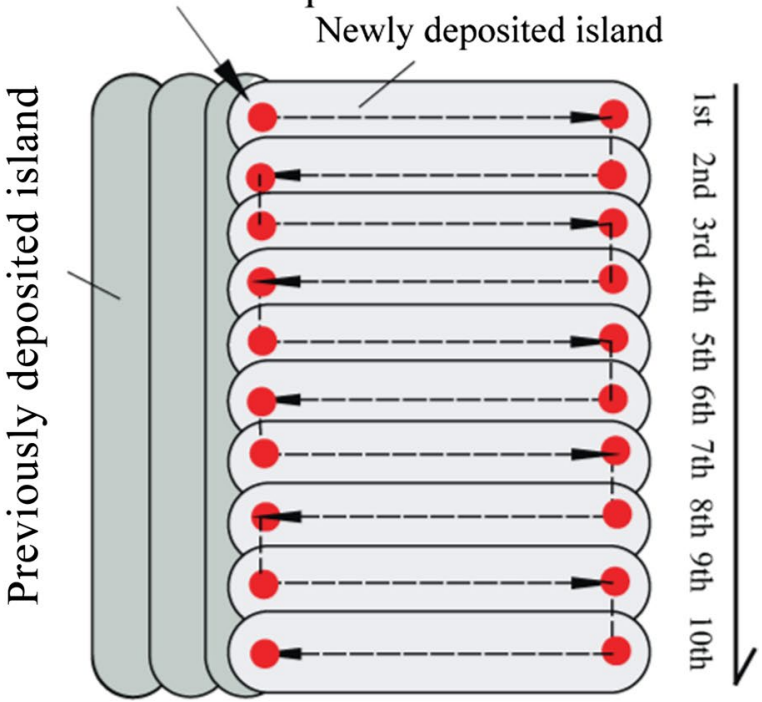

Fig. 6 Overlapping types by different scanning strategies: a conventional successive scanning; $\mathbf{b}$ island scanning
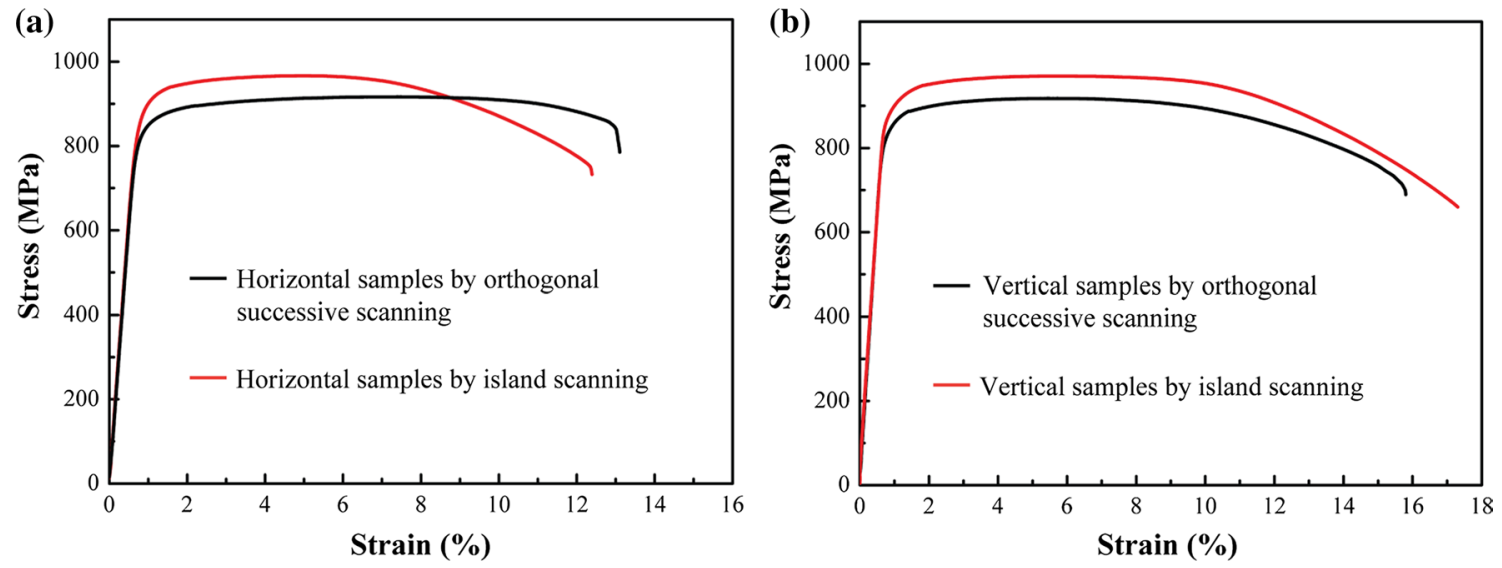

Fig. 7 Tensile stress-strain curves of specimens by different scanning strategies: a horizontal samples; $\mathbf{b}$ vertical samples

Table 1 Tensile properties of specimens by different scanning strategies $\left(\sigma_{0.2}\right.$ : yield strength; $\sigma_{\mathrm{b}}$ : tensile strength; $\delta$ : elongation; $\Psi$ : section shrinkage)

\begin{tabular}{lllll}
\hline & $\sigma_{0.2}(\mathrm{MPa})$ & $\sigma_{\mathrm{b}}(\mathrm{MPa})$ & $\delta(\%)$ & $\Psi(\%)$ \\
\hline Successive horizontal & 917 & 829 & 13.1 & 49.6 \\
Island horizontal & 966 & 890 & 12.2 & 48.4 \\
Successive vertical & 918 & 838 & 15.8 & 51.8 \\
Island vertical & 970 & 917 & 17.2 & 50.4 \\
\hline
\end{tabular}

significantly smaller than that of the sample scanned using orthogonal successive scanning. The pores generated by island scanning at area overlap locations produce cracks during the tensile test, which increase the likelihood of material failure. Yet due to the small grain size and their strong ability to prevent crack growth, the specimens do not break immediately. Unlike the horizontal sample, the vertical sample avoids area overlap in its gauge section when intercepted to avoid concentrated defects [18]. In the case of the inner $\alpha$ grains being finer, tensile strength and elongation in the vertical sample prepared using island scanning exceed that in the sample prepared using orthogonal successive scanning.

To better understand the tensile behavior of these samples, the fracture surfaces of the tested specimens were examined and the results are shown in Fig. 8. The fracture surface consists of a fiber area and shear lip, and a more pronounced necking phenomenon appears, but they show 

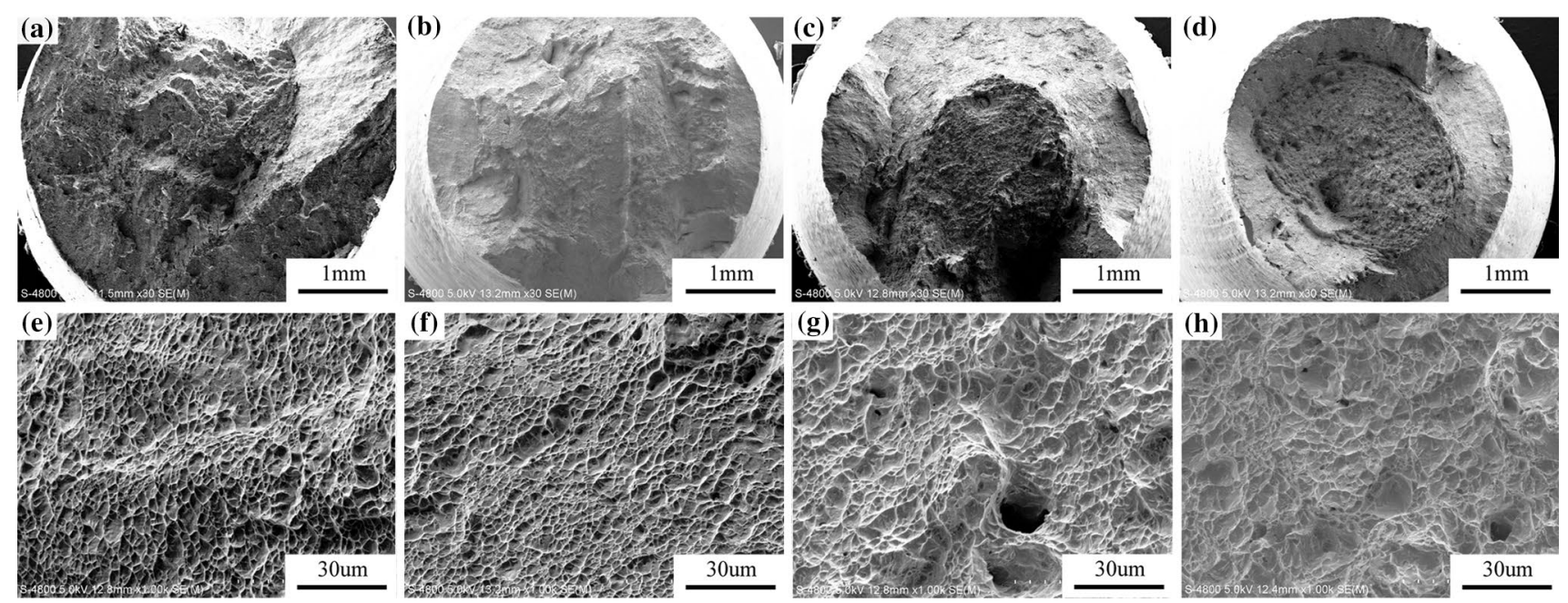

Fig. 8 SEM micrographs of fracture surfaces of specimens by different scanning strategies: a, e horizontal specimen by orthogonal successive scanning; b, f horizontal specimen by island scanning; $\mathbf{c}, \mathbf{g}$

distinct fracture features [19]. As displayed in Fig. 8a, b, the horizontal specimens by two scanning strategies both exhibit ductile and brittle mixtures. Macroscopic deformation near the fracture of the horizontal sample prepared using orthogonal successive scanning is larger, indicating relatively good toughness. Although obvious tearing edges are observed in the fracture surface of the specimen prepared using island scanning, many small, flat cleavage planes are distributed on the fracture surface; therefore, the plasticity of the horizontal specimens prepared using island scanning is lower than that using orthogonal successive scanning, consistent with the tensile property results. As for the vertical specimens shown in Fig. 8c, $\mathrm{d}$, the fracture surfaces both show a cup cone shape, and the macroscopic deformation is quite large. As shown in Fig. 8e-h, compared with dimples on the fracture surface of the horizontal samples, the vertical samples exhibit larger and deeper inner dimples, reflecting good plasticity of the vertical specimens prepared using two scanning strategies.

According to the above results, a basket-weave $\alpha$-phase microstructure characterization appears in both specimens by the two scanning strategies. The $\alpha$ laths inside are thick, short, and distributed in a disordered fashion. This trend creates short slip layers so the cracks cannot be extended in the same direction to cleave the fracture surface. The final specimens are mainly characterized by ductile fractures. As for the many small cleavage planes appearing in the horizontal specimen prepared using island scanning, the planes should be caused by stress concentration and crack extension due to defects in the area overlap. Many small defects appear on the fracture surface in Fig. 8f, supporting the above assumption. However, the vertical vertical specimen by orthogonal successive scanning; $\mathbf{d}, \mathbf{h}$ vertical specimen by island scanning

specimen prepared using island scanning shows good ductility with finer grains, avoiding defect concentration in the overlap.

In conclusion, island scanning can improve the tensile properties of the samples by refining the grains, while the defects introduced substantially reduce sample plasticity. In general, the tensile properties of the samples prepared using island scanning are still higher than the forging standard [20]. However, the microdefects introduced at the area junction can weaken the plasticity of the samples, to further improve plasticity, the hot isostatic pressing process can be applied to reduce internal pores.

\section{Conclusions}

1. The samples subjected to orthogonally successive scanning and island scanning exhibit columnar $\beta$-grain morphology and a basket-weave microstructure. Island scanning significantly refines the grains due to faster cooling during deposition.

2. The relative density of the sample prepared using island scanning is slightly smaller than that of the sample prepared using orthogonal successive scanning due to the concentration of lack-of-fusion pores at the island overlap zone.

3. The ultimate tensile strength and yield strength of the sample prepared using island scanning is better than that prepared by orthogonal successive scanning, while the ductility of the former is weakened due to the concentration of lack-of-fusion pores. 
Acknowledgements This work was financially supported by the Science and Technology Support Program of Jiangsu (Nos. BE2014009-1 and BE2014009-2) and the Key Research and Development Program of Jiangsu (No. BE2015161).

\section{References}

[1] M. Thomas, G.J. Baxter, I. Todd, Acta Mater. 108, 26 (2016)

[2] Z. Zhao, J. Chen, X. Lu, H. Tan, X. Lin, W.D. Huang, Mater. Sci. Eng. A 691, 16 (2017)

[3] L. Xin, H. Yang, C. Jing, Acta Metall. Sin. (Engl. Lett.) 42, 361 (2006)

[4] L.E. Weiss, R. Merz, F.B. Prinz, G. Neplotnik, P. Padmanabhan, L. Schultz, K. Ramaswami, J. Manuf. Syst. 16, 239 (1997)

[5] J. Yao, T. Suo, S.Y. Zhang, F. Zhao, H.T. Wang, J.B. Liu, Y.Z. Chen, Y.L. Li, Mater. Sci. Eng. A 677, 153 (2016)

[6] P. Rangaswamy, M.L. Griffith, M.B. Prime, T.M. Holden, R.B. Rogge, J.M. Edwards, R.J. Sebring, Mater. Sci. Eng. A 399, 72 (2005)

[7] C.L. Qiu, G.A. Ravi, C. Dance, A. Ranson, S. Dilworth, M.M. Attallah, J. Alloys Compd. 629, 351 (2015)

[8] A.J. Sterling, B. Torries, N. Shamsaei, S.M. Thompson, D.W. Seely, Mater. Sci. Eng. A 655, 100 (2016)
[9] P.A. Kobryn, E.H. Moore, S.L. Semiatin, Scr. Mater. 43, 299 (2000)

[10] G. Yang, J.P. Zhou, L.Y. Qin, W. Wang, Trans. China Weld. Inst. 5, $63(2017)$

[11] P.A. Kobryn, S.L. Semiatin, J. Mater. Process. Technol. 135, 330 (2003)

[12] C. Selcuk, Powder Metall. 54, 94 (2011)

[13] H. Yan, L.D. Shen, X. Wang, Z.J. Tian, G.J. Xu, D.Q. Xie, H.X. Liang, Int. J. Adv. Manuf. Technol. 1, 1 (2018)

[14] F.C. Liu, X. Lin, C.P. Huang, M.H. Song, G.L. Yang, J. Chen, W.D. Huang, J. Alloys Compd. 509, 4505 (2011)

[15] H. Paydas, A. Mertens, R. Carrus, J. Lecomte-Beckers, J.T. Tchuindjang, Mater. Des. 85, 497 (2015)

[16] J. Yu, X. Lin, L. Ma, J.J. Wang, X.L. Fu, J. Chen, W.D. Huang, Mater. Sci. Eng. A 528, 1094 (2011)

[17] Y.Z. Zhu, S.Z. Wang, B.L. Li, Z.M. Yin, Q. Wan, P. Liu, Mater. Des. 55, 456 (2014)

[18] Q. Wang, S. Zhang, C.H. Zhang, C.L. Wu, L. Ren, J.Q. Wang, J. Cheng, Acta Metall. Sin. (Engl. Lett.) 31, 19 (2018)

[19] F.Y. Dong, P. Zhang, J.C. Pang, Q.Q. Duan, Y.B. Ren, K. Yang, Z.F. Zhang, Acta Metall. Sin. (Engl. Lett.) 29, 140 (2016)

[20] G.A. Ravi, X.J. Hao, N. Wain, X. Wu, M.M. Attallah, Mater. Des. 47, 731 (2013) 